\title{
Phase II trial on SBRT for unresectable liver metastases: long-term outcome and prognostic factors of survival after 5 years of follow-up
}

Marta Scorsetti ${ }^{1,2}$, Tiziana Comito ${ }^{1}$, Elena Clerici ${ }^{1}$, Ciro Franzese ${ }^{1}$, Angelo Tozzi ${ }^{1}$, Cristina Iftode ${ }^{1}$, Lucia Di Brina ${ }^{1}$, Pierina Navarria', Pietro Mancosu', Giacomo Reggiori ${ }^{1}$, Antonella Fogliata', Stefano Tomatis ${ }^{1}$, Guido Torzilli' ${ }^{2,3}$ and Luca Cozzi ${ }^{1,2,4^{*}}$

\begin{abstract}
Background: The aim of this study was to evaluate long-term efficacy and survival prognostic factors of stereotactic body radiation therapy (SBRT) for un-resectable liver metastases in patients enrolled in a prospective phase II trial.

Methods and materials: 5 -year local control (LC), overall survival (OS), progression free survival (PFS) and toxicity rates were analyzed in patients with un-resectable liver metastases enrolled in a Phase II Trial on liver SBRT, with a prescription dose of 75Gy in 3 consecutive fractions.

Results: A total of 61 patients with 76 lesions were enrolled, with a median follow-up time of 6.1 years. One, three and 5 year $L C$ rates were $94 \pm 3.1 \%, 78.0 \pm 5.9 \%$ and $78.0 \pm 5.9 \%$, without reaching the median LC time. Median OS was 27.6 months and the survival rates were $85.2 \pm 4.5 \%, 31.1 \pm 5.9 \%$ and $18.0 \pm 4.9 \%$ at 1,3 and 5 -year after SBRT, respectively. Univariate analysis showed that favorable primary site (colorectal, breast and gynecological) of metastases $(p=0.001$ ) improved survival. Toxicity was moderate. One patient experienced G3 late chest wall pain, which resolved within 1 year from SBRT. No cases of Radiation Induced Liver Disease (RILD) were detected.

Conclusions: Long-term results of this Phase II study suggest the efficacy and safety of SBRT for un-resectable liver metastases after 5-year of follow up. Selection of cases with positive prognostic factors may improve long-term survival of these oligo-metastastic patients and may confirm the role of SBRT as an effective alternative local therapy for liver metastases.
\end{abstract}

Keywords: Metastatic liver cancer, SBRT, SABR, Volumetric modulated arc therapy

\section{Background}

Early diagnosis, surgical techniques, effective chemotherapy regimens and the introduction of new ablative local therapy have significantly increased quality of life and overall survival of patients with cancer [1]. Multidisciplinary management of cancer led to an increased accrual

\footnotetext{
* Correspondence: luca.cozzi@humanitas.it

${ }^{1}$ Radiotherapy and Radiosurgery Department, Humanitas Cancer Center, Humanitas Clinical and Research Center, Via Manzoni 56, 20089 Rozzano, Milan, Italy

${ }^{2}$ Department of Biomedical Sciences, Humanitas University, Via Manzoni 113, 20089 Rozzano, Milan, Italy

Full list of author information is available at the end of the article
}

of patients with few organ-confined metastases, who can experience long-term survival [2].

Liver is one of the most common sites of oligometastatic disease from several cancers, such as colorectal, breast and lung cancer. When metastatic disease from colorectal cancer (CRC) is confined to the liver, surgical resection represents the standard of care and it is associated with a better prognosis [3-6]. Approximately $70-90 \%$ of liver metastases are however un-resectable and a safe and effective alternative therapeutic option is necessary.

Minimally invasive local approaches were introduced as an alternative to surgery, including radiofrequency ablation (RFA) and microwave ablation (MWA). Nevertheless, 
these techniques have limits, mostly related to lesion size and location (lesions greater than $3-4 \mathrm{~cm}$ in diameter or located in proximity of major blood vessels, biliary tract, gallbladder or just beneath the diaphragm) $[7,8]$.

An increasing number of prospective trials on liver stereotactic body radiation therapy (SBRT) were published, with encouraging results in terms of local control (LC), toxicity and overall survival (OS) [9-17]. Relevant factors affecting the efficacy of SBRT were identified and included tumor size, prescription dose and histology [12, 18-23].

The aim of this study was to analyze long-term results of liver SBRT and to confirm the safety and the efficacy of this ablative therapy in the treatment of these oligometastatic patients with a complete 5-year follow-up.

\section{Methods and materials}

\section{Patient cohort}

Phase 2 prospective trial on SBRT for un-resectable liver oligo-metastases was approved by the internal ethical committee of Humanitas Cancer Center Hospital in 2010. Inclusion criteria of this observational study were described in previous publications [16, 17] and summarized in Table 1.

\section{SBRT technique}

SBRT technique and assessment of efficacy and morbidity were described in previous publications $[16,17]$. Patients were immobilized with a thermoplastic body mask, including a Styrofoam block for abdominal compression. A contrast-free and triple-phases contrast-enhanced computed tomography $(\mathrm{CT})$ scan were acquired for all patients. The 4-dimensional CT (4D-CT) imaging was performed in patients with respiratory excursion greater than $5 \mathrm{~mm}$. In most of patients, planning CT images were co-registered with magnetic resonance imaging (MRI) or

Table 1 Inclusion criteria

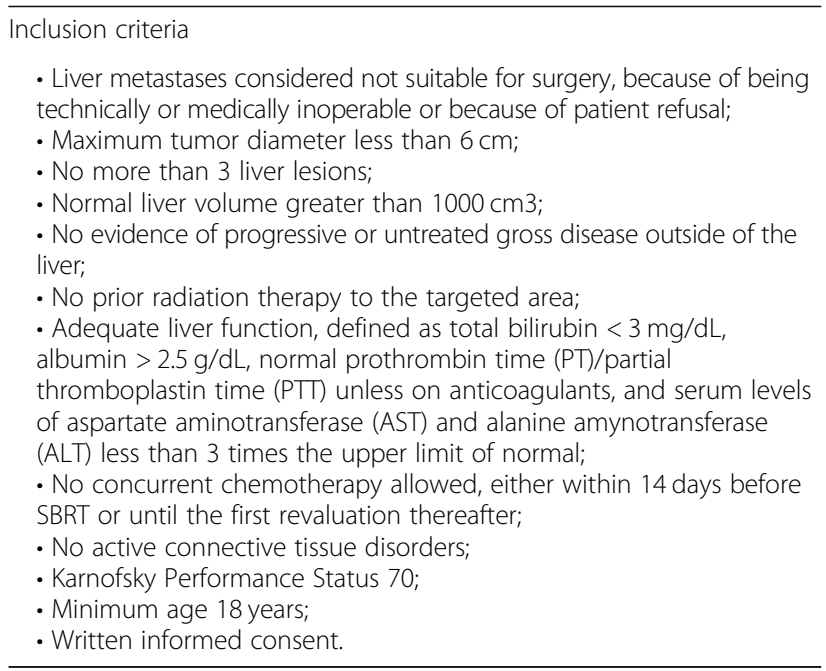

positron emission tomography (PET) to better identify the target volume. The clinical target volume (CTV) was defined as equal to the gross tumor volume (GTV). In all patients who underwent 4D-CT scan, an internal target volume (ITV) was defined as the envelope of all CTVs in the different respiratory phases. The planning target volume (PTV) was generated from either the CTV or the ITV by adding an isotropic margin of $5 \mathrm{~mm}$ from ITV or of $7-10 \mathrm{~mm}$ in the cranial-caudal axis and $4-6 \mathrm{~mm}$ in the anterior-posterior and lateral axes from CTV. The plan objective was to cover at least $98 \%$ of the CTV (ITV) volume with $98 \%$ of the prescribed dose $\left(\mathrm{V}_{98 \%}=98 \%\right)$ and for the PTV to cover $95 \%$ of the volume with $95 \%$ of the dose $\left(\mathrm{V}_{95 \%}=95 \%\right)$. Prescription dose was 75 Gy in 3 consecutive daily fractions of $25 \mathrm{~Gy}$ each, prescribed as the mean dose to PTV. An individualized dose scheme, where the prescription dose might be reduced in a step-wise manner, was employed to respect dose constraints for organs at risk (OARs). Simulation phase, treatment planning, dose prescription, dose constraints for OARs and treatment delivery were summarized in Table 2 . No specific constraints were applied in terms of conformality index. Dose gradient was controlled by means of the normal tissue objective tool as suggested in the table. Treatment was delivered in free breathing on a Varian TrueBeam linear accelerator using a 10 MV Flattening Filter Free beam with a maximum nominal dose rate of $2400 \mathrm{MU} /$ minute with the RapidArc technique. Two coplanar partial arcs were used for the plans as a class solution. Three dimensional image guided radiation therapy (IGRT) was performed before each daily session to check patient and tumor position by means of cone beam CT (CBCT). In most of patients we identified and matched the bone land marks and soft tissue matching from the CBCT data with respect to the planning CT. In some patients who had previous surgery, surgical clips were used as markers to enhance the quality of the task.

\section{Trial end points and response assessment}

Primary end-point was to assess in field LC. Secondary end points were to define radiation treatment-related toxicity and OS.

Tumor response was defined according to European Organization for Research and Treatment of Cancer Response Evaluation Criteria In Solid Tumors (EORTC-RECIST) 1.1 [24]. After SBRT, physical examination and basal blood chemistry analyses were performed 21 days after and then every 2 months. Radiological restaging was performed every 3 months for 3 years and every 6 months for the following 2 years, if patients were disease-free. Acute and late toxicity were scored by the Common Terminology Criteria for Adverse Events 3.0.

Considering local control as primary endpoint, the study was designed to exclude a local control $<60 \%$ with 
Table 2 Treatment planning and delivery characteristics

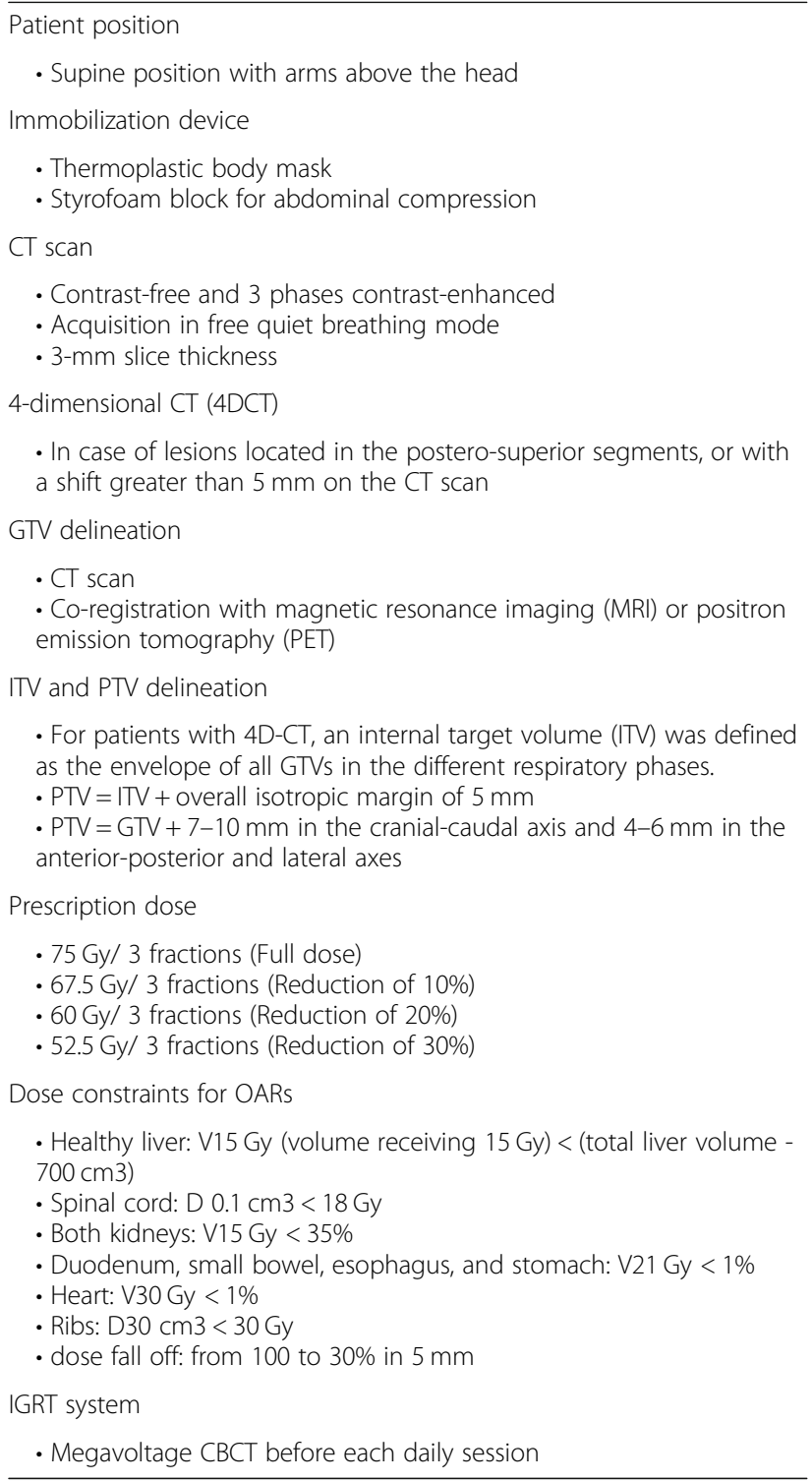

an $\alpha$-error of $5 \%$ and to demonstrate a LC $>80 \%$ with a power of $90 \%$. This required the enrollment of 44 patients, with at least 33 disease local control was necessary. Actuarial LC, OS and PFS curves were generated by using the Kaplan-Meier method. Log rank test was used for group comparison. Survival was measured from the end of SBRT. Univariate analysis was used to correlate morphologic and clinical factors to LC, OS and PFS and statistical significance was accepted for $p$-values of $<0.05$. A multivariate analysis was not performed due to low number of events in this cohort of patients. All analyses were performed using STATA version 13 software.

\section{Results}

Sixty-one patients with 76 liver metastases were treated with SBRT between February 2010 and September 2011 in this phase II study. Baseline patients and treatment characteristics are previously described in a published paper on preliminary results $[16,17]$. Twenty-eight patients $(45.9 \%)$ received liver-directed therapy before the SBRT treatment. Particularly, 21 patients were treated with hepatic surgery, 2 with RFA and 5 with both. Twenty-nine patients (47.5\%) were treated for CRC liver metastases, 11 (18\%) for breast cancer hepatic lesions and 7 (11.5\%) for gynecological cancer liver metastases. Other primary tumors were exocrine pancreas in 5 patients, lung in 3 patients, kidney in 2 patients, melanoma in 2 patients and head and neck in 2 patients. Most of lesions $(82 \%)$ were treated with the full prescription dose of $75 \mathrm{~Gy}$ in 3 fractions, $8 \%$ with $67.5 \mathrm{~Gy}$ (reduction dose of $10 \%$ ), $5 \%$ of lesions with $60.0 \mathrm{~Gy}$ (reduction dose of $20 \%$ ) and $5 \%$ with $52.5 \mathrm{~Gy}$ (reduction dose of $30 \%$ ). At the time of SBRT, 21 (34.4\%) patients presented with stable extrahepatic disease and 40 (65.6\%) had no evidence of other sites of metastases.

All clinical cases were discussed by a liver multidisciplinary board including an expert hepatic surgeon. According to the international expert consensus [25] for all patients was defined the un-resectable status, considering the presence of one or more of following main factors.

\section{Extrahepatic disease}

Twenty one (34.4\%) patients presented un-resectable extra-hepatic disease at the time of SBRT

\section{Prior hepatic surgery}

Twenty one (34.5\%) patients were pre-treated with liver surgery and a second resection was not feasible

\section{Age and comorbidity}

Twenty four (39\%) are elderly patients and $40 \%$ of patients presented relevant comorbidities which did not allow the use of chemotherapy (16.4\%) or the continuation beyond the first line of systemic therapies (24.6\%)

\section{Lesions site}

Thirty one (51\%) patients presented central lesion, located close to the central biliary tract $(\mathrm{HBC})$ and portal vein (PV).

According to the literature [26, 27] central lesions were defined as lesions located outside of the central liver zone, defined as a 2-cm expansion around the course of the portal vein contoured to its bifurcation in the liver. We treated 31 (51\%) patients with a total of 40 (53\%) central lesions with a prescription doses of 52.5Gy (4 lesions), 60Gy (6 lesions), 67.5Gy (60 lesions) and 75.0Gy (24 lesions).

The median FUP was 6.1 years (range 3-82 months). 
At the time of analysis, 10 patients (16\%) are alive, without progression of disease. Most of the deceased patients (94\%) died for progression of disease. The remaining patients died of non-cancer related causes. After SBRT, 12 (20\%) patients presented intrahepatic progression only (of these 8 (13\%) with extra-field progression and 4 (6\%) with extra- and in-field progression), $17(28 \%)$ patients showed extra-hepatic progression only and $16(26 \%)$ patients presented both intra-hepatic and extra-hepatic progression of disease. Median OS was 27.6 months (95\%CI: $23.2-34.8$ ) and a range of $2.1-81.5$ months and the survival rates were $85.2 \pm 4.5 \%, 31.1 \pm$ $5.9 \%$ and $18.0 \pm 4.9 \%$ at 1,3 and 5 year after SBRT, respectively. Figure 1a showed the Kaplan-Meier curve for OS. At univariate analysis, survival was significantly better in patients with favorable primary disease (colorectal, breast and gynecological cancer) compared to other primary sites of metastases $(p=0.001)$, as showed in Fig. 1b. The OS was independent of tumor size of liver metastases $(p=0.47)$ and the presence of stable extra-hepatic disease did not correlate to OS $(p=0.88)$. Patients with a single lesion presented with a 5 year OS of $28.6 \%$, compared to $14.9 \%$ for patients with $2-3$ lesion, however this difference was not significant $(p=0.22)$. Patients pre-treated with more than 2 line of chemotherapy (CHT) had a 5 year OS of $10.9 \%$ compared to $30.4 \%$ for those who received 0 or 1 line of CHT before the SBRT, but also this difference is not significant $(p=0.10)$. Table 3 summarized the univariate analysis results for OS.

The LC rates at one, 3 or 5 were $94 \pm 3.1 \%, 78.0 \pm 5.9 \%$ and $78.0 \pm 5.9 \%$, respectively, the median LC time was not reached while the estimated mean LC time was 74.8 \pm 3.9 months (95\%CI: 67.1-82.5 months). Although cancer type was not related to local relapse, a sub group of breast and gynecological cancer patients had a very favorable $\mathrm{LC}$ rates of $86.1 \pm 4.7 \%$ and $86.1 \pm 5.6 \%$ at 5 -year. For CRC liver metastases 5-year LC rate was $75 \%$.
Previous local ablative therapies were borderline significant for local control $(p=0.06)$. Liver metastases pre-treated with surgery or RFA presented with a 5-year LC of $65.8 \pm 4.6$, compared to hepatic lesions without prior local ablative treatments which had a LC rate at 5 -year of $87.1 \pm 5.8 \%$. Patients with tumor size higher than $3 \mathrm{~cm}$ presented a local control at 5-year comparable to patients treated for smaller hepatic lesions $(81.9 \%$ vs $77.2 \% ; p<0.60)$. Figure $2 \mathrm{~b}$ showed a case of local control in 1 liver lesion treated with 75Gy in 3 fraction. Acute toxicity has been explained in a published preliminary report [16]. Analysis at 3 and 5-year confirmed the toxicity profile of our fractionation anticipated in the preliminary studies. As previously reported, only 1 patient experienced G3 late toxicity at 6 months, resulting from severe chest wall pain, which regressed within 1 year after treatment. In 2 patients G2 late toxicity was detected for appearance of moderate chest wall pain at 5 and 7 months, respectively. Concerning the healthy liver involvement, from the planning data, the mean liver dose was $12.7 \pm 5.1 \mathrm{~Gy}$ and V15Gy was $1050 \pm 318 \mathrm{~cm}^{3}$.

\section{Discussion}

To date, the role of SBRT as ablative therapy of liver metastases is not consolidated and only recently, ESMO guidelines [28] added SBRT in the toolbox of local therapy for selected patients with CRC liver metastases unsuitable for surgery or RFA/MWA. All this is mainly related to the lack of long-term results from prospective and randomized trials. This report summarized the results of a phase II trial with more than 5-year follow-up for patients treated with high dose SBRT. The rational and the early results for the very high dose regimen proposed are summarized in our own earlier works [16, 17, 29].

Concerning treatment margins, the present study described the data from the original protocols which required relative large expansions from the CTV or ITV. With the current practice and with the knowledge that

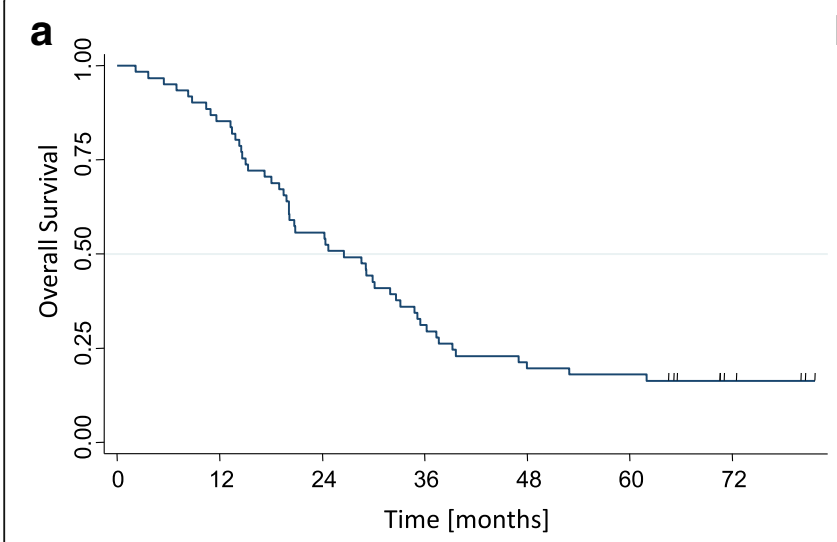

b

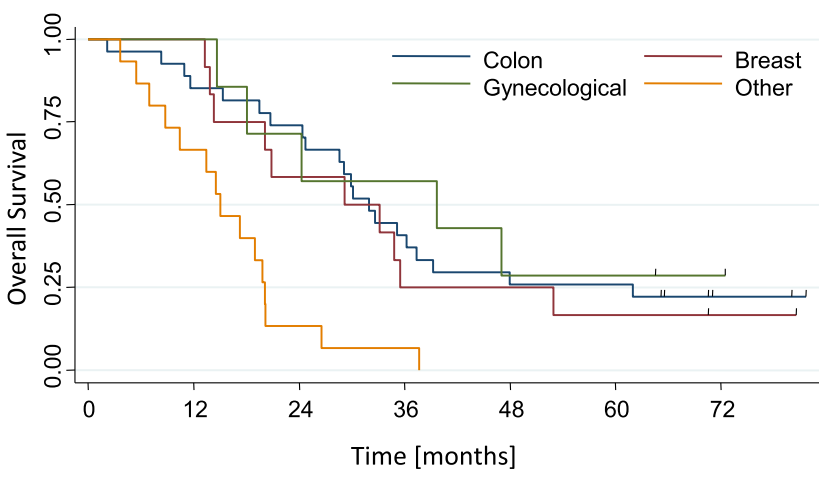

Fig. 1 a Overall survival (OS); b OS by primary tumor 
Table 3 Univariate analysis for overall survival and local control

\begin{tabular}{|c|c|c|c|c|c|c|c|}
\hline \multirow[t]{2}{*}{ Covariate categories } & \multirow[t]{2}{*}{$N$} & \multicolumn{2}{|l|}{ OS (\%) } & \multirow[t]{2}{*}{$p$} & \multicolumn{2}{|l|}{ LC (\%) } & \multirow[t]{2}{*}{$p$} \\
\hline & & 3 years & 5 years & & 3 years & 5 years & \\
\hline \multicolumn{8}{|l|}{ Age, years } \\
\hline$>70$ & 24 & $25.0 \pm 8.8$ & $16.7 \pm 7.6$ & \multirow[t]{2}{*}{0.57} & $64.2 \pm 4.7$ & $64.2 \pm 5.9$ & \multirow[t]{2}{*}{0.71} \\
\hline$<70$ & 37 & $35.1 \pm 7.8$ & $18.9 \pm 6.4$ & & $82.3 \pm 4.4$ & $82.3 \pm 5.6$ & \\
\hline \multicolumn{8}{|l|}{ Gender } \\
\hline Male & 35 & $20.6 \pm 6.9$ & $11.8 \pm 5.5$ & \multirow[t]{2}{*}{0.01} & $78.1 \pm 4.3$ & $78.1 \pm 5.4$ & \multirow[t]{2}{*}{0.97} \\
\hline Female & 26 & $44.4 \pm 9.6$ & $25.9 \pm 8.4$ & & $81.8 \pm 4.1$ & $81.8 \pm 5.4$ & \\
\hline \multicolumn{8}{|l|}{ Cancer type } \\
\hline Colorectal & 29 & $41.1 \pm 7.5$ & $27.3 \pm 8.1$ & \multirow[t]{4}{*}{$<0.001$} & $75.3 \pm 4.7$ & $75.3 \pm 5.7$ & \multirow[t]{4}{*}{0.47} \\
\hline Breast cancer & 11 & $33.0 \pm 7.3$ & $20.1 \pm 6.9$ & & $86.8 \pm 4.8$ & $86.8 \pm 5.7$ & \\
\hline Gynecological cancer & 7 & $56.8 \pm 6.9$ & $27.8 \pm 7.3$ & & $86.1 \pm 4.7$ & $86.1 \pm 5.6$ & \\
\hline Other & 14 & $10.2 \pm 7.2$ & - & & $80.0 \pm 4.4$ & - & \\
\hline \multicolumn{8}{|l|}{ Number of metastases } \\
\hline 1 & 48 & $42.8 \pm 13.2$ & $28.6 \pm 12.1$ & \multirow[t]{2}{*}{0.22} & $82.0 \pm 4.5$ & $82.0 \pm 5.4$ & \multirow[t]{2}{*}{0.28} \\
\hline $2-3$ & 13 & $27.7 \pm 6.5$ & $14.9 \pm 5.2$ & & $67.3 \pm 4.7$ & $67.3 \pm 5.6$ & \\
\hline \multicolumn{8}{|l|}{ Size of metastases } \\
\hline$<3 \mathrm{~cm}$ & 32 & $28.1 \pm 7.9$ & $15.6 \pm 6.4$ & \multirow[t]{2}{*}{0.46} & $77.2 \pm 4.3$ & $77.2 \pm 5.5$ & \multirow[t]{2}{*}{0.60} \\
\hline$>3 \mathrm{~cm}$ & 29 & $34.4 \pm 8.8$ & $20.7 \pm 7.5$ & & $81.9 \pm 4.3$ & $81.9 \pm 5.9$ & \\
\hline \multicolumn{8}{|l|}{ Timing of metastasis } \\
\hline Synchronous & 24 & $29.3 \pm 6.5$ & $17.0 \pm 5.5$ & \multirow[t]{2}{*}{0.93} & - & & \multirow[t]{2}{*}{-} \\
\hline Metachronous & 37 & $32.2 \pm 8.1$ & $19.3 \pm 6.9$ & & & & \\
\hline \multicolumn{8}{|l|}{ Time since diagnosis, mo } \\
\hline$\leq 12$ & 35 & $31.4 \pm 7.8$ & $17.1 \pm 6.4$ & \multirow[t]{2}{*}{0.91} & - & & \multirow[t]{2}{*}{-} \\
\hline$>12$ & 26 & $30.8 \pm 9.0$ & $19.2 \pm 7.7$ & & & & \\
\hline \multicolumn{8}{|l|}{ Prior local therapy } \\
\hline Yes & 27 & $33.3 \pm 9.1$ & $11.1 \pm 6.1$ & 0.54 & $65.8 \pm 4.6$ & $66.7 \pm 5.7$ & 0.06 \\
\hline No & 34 & $29.4 \pm 7.8$ & $23.5 \pm 7.3$ & & $87.1 \pm 4.8$ & $87.1 \pm 5.8$ & \\
\hline Pre-SBRT chemotherapy & & & & & & & \\
\hline 0-1 schedule & 23 & $43.5 \pm 10.3$ & $30.4 \pm 9.6$ & 0.10 & - & & - \\
\hline 2-3-4 schedules & 38 & $23.7 \pm 6.9$ & $10.5 \pm 5.0$ & & & & \\
\hline Extrahepatic disease & & & & & & & \\
\hline Yes & 21 & $33.3 \pm 10.3$ & $14.3 \pm 7.6$ & 0.88 & - & & - \\
\hline No & 40 & $30.0 \pm 7.2$ & $20.0 \pm 6.3$ & & & & \\
\hline
\end{tabular}

well-done abdominal compression, eventually associated to some additional breath control (e.g. breath hold) might allow to further reduce the margins and, consequently, to further protect the healthy fraction of the liver. This option was not part of the current investigation.

Table 4 summarizes the long-term outcome data of the few published SBRT trials with more than 40 enrolled patients and with a median follow-up longer than 2 years. The study by McPartlin [30], reported a 2 and 4-year LC rates of 49.8 and $26.2 \%$, respectively for patients treated with 33 to $57 \mathrm{~Gy}$ in 6 fractions.
These results are worse than surgery and thermo-ablation and dose escalation is needed to improve sustained local control, as stated by the same authors. Similar conclusions were confirmed by Klement and by Joo, asserting that the use of ablative prescription dose is a crucial starting point to make SBRT a valid therapeutic option [31-33]. Joo [31] reported 2-year LC rates of 52,83 , and $89 \%$ using a biological equivalent dose (BED) $\leq 80$ Gy (group 1), 100-112Gy (group 2 ), and $\geq 132 \mathrm{~Gy}$ (group 3), respectively. The authors concluded that better LC can be expected if higher doses are applied. 


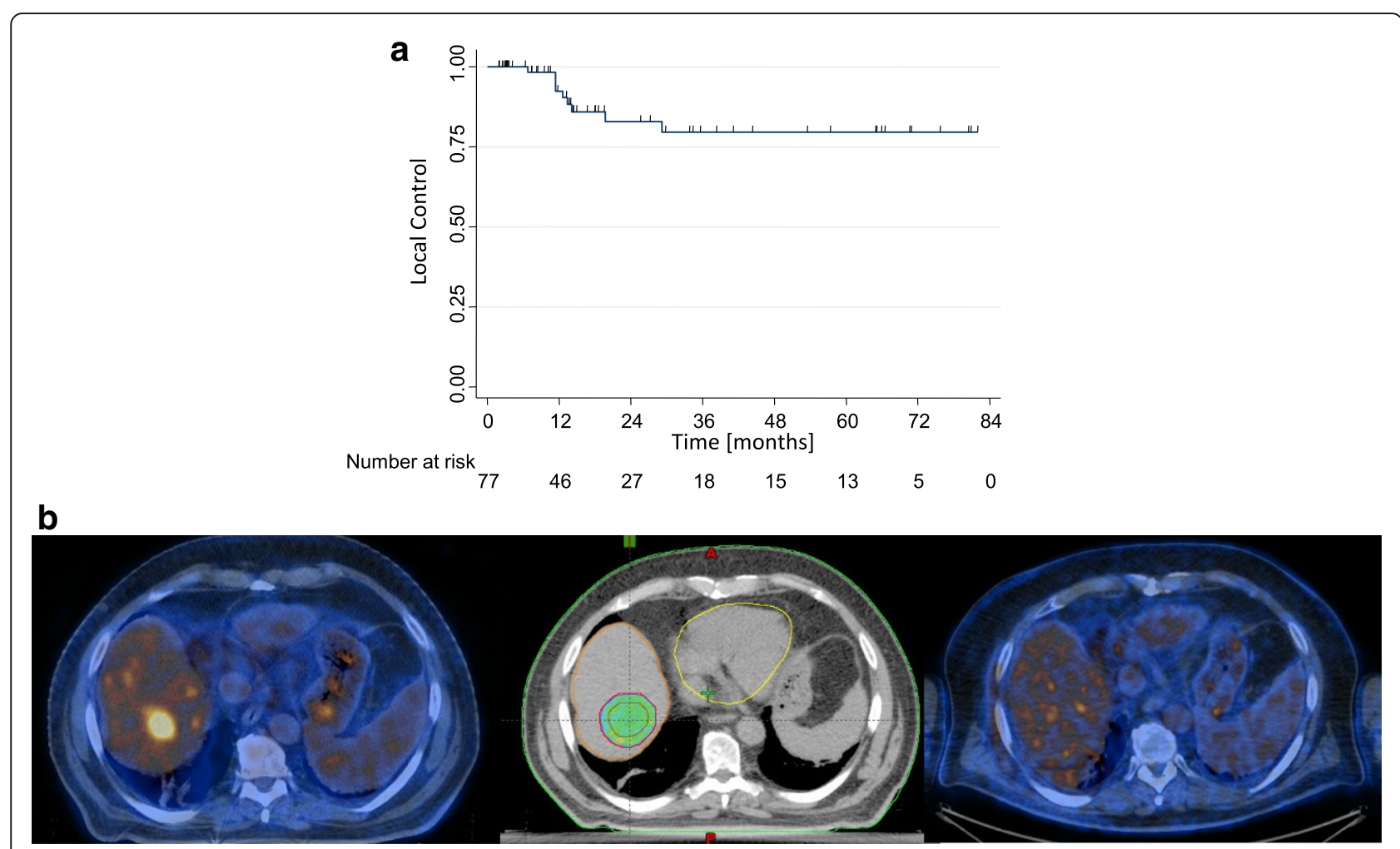

Fig. 2 a Local control; b an example of a patient with complete response at 6 months from treatment

Klement [32, 33] suggested the use of doses greater than 3x17Gy and suggested to deliver a BED of $209 \pm$ 67 Gy to achieve a 2 -year LC rate of $90 \%$ in patients with no prior chemotherapy and $286 \pm 78 \mathrm{~Gy}$ when chemotherapy was administered. The authors confirmed also the strong influence of histology and pre-treatment chemotherapy on LC, concluding that metastases from breast cancer respond better to SBRT compared to other histology types and that increased radiation resistance of CRC metastases may rather reflect the higher resistance after pre-SBRT chemotherapy. These innovative results could improve patient selection to liver SBRT and its efficacy, even though more prospective long-term data are needed.

The current prospective phase II study could therefore contribute to this search for evidence.

The fractionation employed in our trial escalates the dose with a BED ranging from 144.38Gy to 262.5Gy. Our results confirmed the safety and efficacy of ablative doses with a very high and sustained LC rate of $78 \%$ and with a very low toxicity profile after more than 5 years of follow up. Considering the suggestions of previously cited studies, our results showed also the effectiveness in disease progression control even in those unfavorable patients with bigger lesions (diameter $>3 \mathrm{~cm}$ ), radio-resistant histology such as CRC and/or heavily pre-treated with systemic and local therapies.
The size of the lesions is a relevant factor affecting local control of ablative radiotherapy. Correlation between LC and lesion size higher than $3 \mathrm{~cm}$ was demonstrated by Rusthoven in a phase II trial on liver SBRT, using a dose prescription of 60Gy in 3 fractions [12]. Dose-response and size-response relationships were confirmed also in the recent report by McPartlin [30], with a 4 -year local control rate of $26 \%$, correlated with the GTV. Long-term results using the fractionation of $75 \mathrm{~Gy}$ in 3 fractions showed that a promising LC can be also obtained in bigger lesions with a diameter ranging from 3 to $6 \mathrm{~cm}$, with a 5 -year LC rate of $81 \%$ as shown in the current study.

The high local response rate was not significantly influenced by any factors in our experience and CRC metastases presented a competitive LC rate at 5 years of $75 \%$, although most of these patients were unfavorably selected. More than $50 \%$ of patients, indeed, received liver-directed therapy prior to SBRT and $86 \%$ of CRC patients were heavily pre-treated with systemic therapies before radiation treatment.

While the role of previous systemic treatments in inducing radio-resistance has been widely explained by Klement [32], the effect of previous local therapies is being studied.

In the last decades, the growth of cancer stem cells (CSC) in the site of previously local-treated 


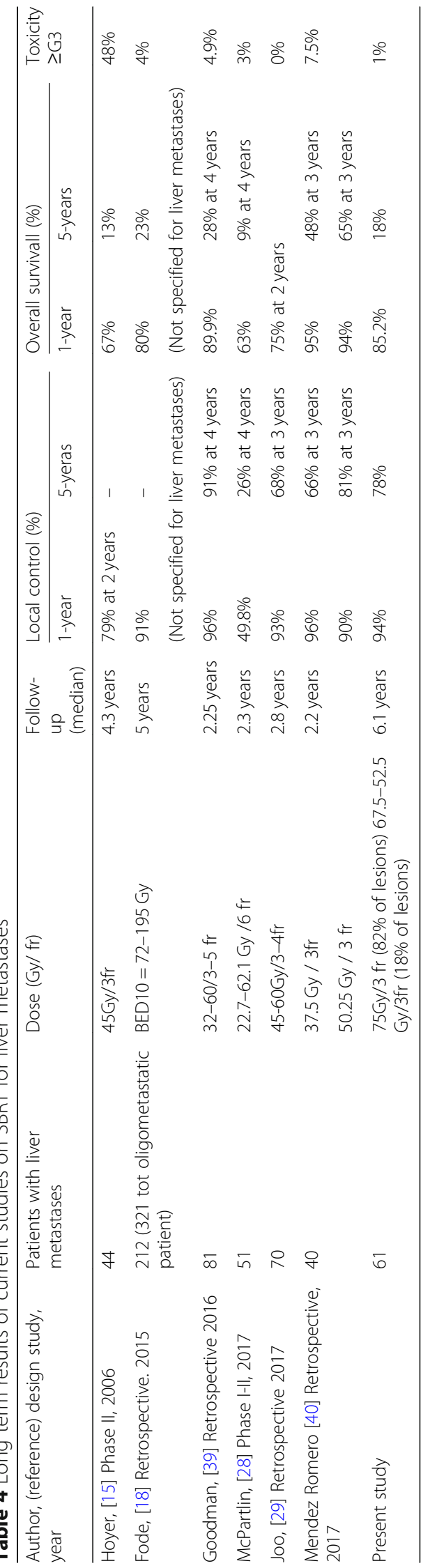


tumors has been demonstrated. CSC seems to have the ability to self-renewal, to generate heterogeneous progeny and to self-divide unlimitedly. Considering these characteristics, CSCs seem to correlate to chemo- and radio-resistance development [35]. In our experience previous local ablative therapies were borderline significant for local control. This result could be explained by considering the limited number of enrolled patients in this trial and future studies on larger series could be needed to confirm this correlation.

Critical analysis of LC data confirmed the suggestion from Klement about a better response to SBRT of breast cancer liver metastases, with an optimal 5-year LC rate of $87 \%$.

Primary tumor and previous therapies are crucial factors influencing the OS also. The efficacy of local ablative therapies as potentially curative for oligometastatic patients is consolidated for surgery of CRC liver disease [25, 34, 36-38], but it is debated for non-CRC liver metastases [39-42].

In a multicenter phase I/II study on patients treated with SBRT for liver metastases, Rusthoven [12] showed a 2 -year OS of $30 \%$. In this prospective experience, OS was better in patients with favorable cancer types (breast, CRC, sarcomas and renal cancer) than in patients with less favorable tumor types (lung, ovarian, and non-CRC gastrointestinal cancers), with a median OS of 32 months versus 12 months, respectively. These results promoted the efficacy of hepatic metastases ablation also for selected non-CRC liver metastases.

Although our study is not comparative and the primary endpoint is not OS, results show a benefit for the use of SBRT in the multidisciplinary management of oligometastatic patients with inoperable liver metastases not only from $\mathrm{CRC}$, but also from other favorable primary disease, such as breast and gynecological cancers. In CRC liver metastases, indeed, our fractionation allowed an encouraging OS rate of $41 \%$ at 3 year and $27 \%$ at 5 year. Similar positive results were also achieved for patients with gynecological cancer-derived liver disease, with 3 and 5 year OS of 57 and 28\%, respectively. Patients irradiated with breast cancer derived liver metastases showed a lower survival with a 3-5 year OS rates of $33-20 \%$, respectively.

This apparently negative outcome could be related to an unfavorable selection of these patients. Indeed, patients with liver metastases from breast cancer, were pretreated with more systemic therapy before SBRT than those patients with other histological types. All 11 (100\%) breast cancer patients received CHT and 8 (73\%) patients were pretreated with more than 3 lines of systemic therapy. In this subgroup of patients, therefore, SBRT was more delayed.
These data confirmed that liver oligo-metastatic patients are often heavily pretreated and are unsuitable for the following systemic or local therapy at the time of referral for SBRT. As shown by Fode [19], the use of SBRT allows to decrease tumor progression in this setting of oligo-metastatic patients, with an encouraging 5-year OS rate of $23 \%$, comparable to our result.

In our trial, all patients were unsuitable for surgery and RFA, $87 \%$ of patients received chemotherapy pre-SBRT and about $40 \%$ were heavily pre-treated with more than 3 lines of systemic therapy before SBRT. Supportive care remained the only therapeutic option for most of these patients, with an expected OS rate likely lower than $23 \%$ at 5 -year [19].

Analyzing the current literature data on oligometastatic patients treated with ablative therapy, other prognostic factors for OS have been also identified beyond those of our study. A possible reason for this data could be given by patient selection.

All patients were enrolled in our trial according to restrictive inclusion criteria. They were defined by considering the importance of same prognostic factors suggested by surgical experiences. Since 1999, Fong showed the significant correlation between OS and specific variables, such as small number of lesions, size $<5 \mathrm{~cm}$, absent or controlled extrahepatic disease, in patients with CRC liver metastases, treated with hepatic surgery [36]. Selection of patients at the time of enrollment, therefore, could justify why these variables do not improve OS in our experience. This remark seems to be confirmed by Chang in a pooled analysis on SBRT for CRC liver metastases [17]. In this experience, which employed inclusion criteria similar to our trial, number of lesions (1 vs $2-4)$, lesion size and active non-liver disease were not correlated with OS, as well.

The need to identify simple predictors of outcome, an important task of state-of-the-art research, was investigated by Mazzola [43]. The authors proposed the use of diagnostic signatures from PET imaging as predictors of local response in liver metastases. These included pre-SBRT SUV-max and SUV-mean. Considering the relatively low sample size, our long term results suggest that patients selected by favorable primary disease, number, size and site of liver metastases and controlled extrahepatic disease, could benefit from high dose of SBRT also in terms of OS.

The data presented in our report refer to linac based SBRT. The use of alternative delivery modalities, like, e.g., robotic devices as CyberKnife has been investigated. A review from Ihnat [44] showed that the most frequently treated are for less than 5 metastases with a maximum of $6 \mathrm{~cm}$ and no extrahepatic disease. In general, severe toxicity is rare and local control range from 70 to $100 \%$ at 1 year and from 60 to $90 \%$ at 2 years. 
As a final remark, it should be advised that, thy type of very high-dose stereotactic regime should be carried out in appropriately qualitied and experienced centers and that sufficient quality assurance procedure are enforced to guarantee the safety of the treatments.

\section{Conclusions}

The 5-year results confirmed the role of high-dose SBRT in the treatment of liver metastases with diameter $>3$ $\mathrm{cm}$, which are often unsuitable for other effective local ablative therapy. Prognostic factors identified suggested the relevant role of cases selection and the timing of SBRT in the therapeutic pathway of these patients.

\section{Abbreviations}

4D-CT: Four dimensional computed tomography; BED: Biological equivalent dose; CHT: Chemotherapy; Cl: Confidence interval; CRC: Colorectal cancer; CSC: Cancer stem cells; CT: Computed tomography; CTV: Clinical target volume; EORTC: European Organization for Research and Treatment of Cancer; ESMO: European society for medical oncology; GTV: Gross tumor volume; IGRT: Image guided radiation therapy; ITV: Internal target volume; LC: Local control; MRI: Magnetic resonance imaging; MU: Monitor unit; MV: Mega voltage; MWA: Microwave ablation; OAR: Organ at risk; OS: Overall survival; PET: Positron emission tomography; PFS: Progression free survival; PTV: Planning target volume; RECIST: Response Evaluation Criteria In Solid Tumors; RFA: Radiofrequency ablation; RILD: Radiation induced liver disease; SBRT: Stereotactic body radiation therapy; SUV: Standardized uptake value

\section{Acknowledgements}

None.

\section{Funding}

Not applicable.

\section{Availability of data and materials}

The datasets used and/or analyzed during the current study are available from the corresponding author on reasonable request. Requests should be addressed to the corresponding author.

\section{Authors' contributions}

LC, MS and TC. PM, GR, AF, ST, performed the planning, TC, ST and LC performed all the analysis and drafted the manuscript. AT, CI, EC, LDB, CF, PN, TC, GT performed the clinical analysis and related activities. All authors reviewed, amended and approved the final version of the manuscript.

\section{Ethics approval and consent to participate}

All procedures performed in studies involving human participants were in accordance with the ethical standards of the institutional and/or national research committee and with the 1964 Helsinki declaration and its later amendments or comparable ethical standards. The Humanitas Ethical Committee approved the study. Informed consent to participate in the treatment was obtained from each patient at admission.

\section{Consent for publication}

Not applicable.

\section{Competing interests}

L. Cozzi acts as Scientific Advisor to Varian Medical Systems and is Clinical Research Scientist at Humanitas Cancer Center. All other co-authors declare that they have no competing interests.

\section{Publisher's Note}

Springer Nature remains neutral with regard to jurisdictional claims in published maps and institutional affiliations.

\section{Author details}

${ }^{1}$ Radiotherapy and Radiosurgery Department, Humanitas Cancer Center, Humanitas Clinical and Research Center, Via Manzoni 56, 20089 Rozzano, Milan, Italy. ${ }^{2}$ Department of Biomedical Sciences, Humanitas University, Via Manzoni 113, 20089 Rozzano, Milan, Italy. ${ }^{3}$ Department of Hepato-biliary Surgery, Humanitas Clinical and Research Center, Via Manzoni 56, 20089 Rozzano, Milan, Italy. ${ }^{4}$ Radiotherapy and Radiosurgery Department, Humanitas Research Hospital and Cancer Center, Via Manzoni 56, 20089 Milan, Rozzano, Italy.

Received: 13 September 2018 Accepted: 16 November 2018 Published online: 26 November 2018

\section{References}

1. De Santis CE, Lin CC, Mariotto $A B$, Siegel RL, et al. Cancer treatment and survivorship statistics, 2014. CA Cancer J Clin. 2014;64:252-71.

2. Weichselbaum RR, Hellman S. Oligometastases revisited. Nat Rev Clin Oncol. 2011:8:378-82.3

3. Wei AC, Greig PD, Grant D, et al. Survival after hepatic resection for colorectal metastases: a 10-year experience. Ann Surg Oncol. 2006;13:668-76.12.

4. Fong $Y$, Fortner J, Sun RL, et al. Clinical score for predicting recurrence after hepatic resection for metastatic colorectal cancer: analysis of 1001 consecutive cases. Ann Surg. 1999;230:309-18 13.

5. Tomlinson JS, Jarnagin WR, Dematteo RP, et al. Actual 10-yearsurvival after resection of 514 colorectal liver metastases defines cure. J Clin Oncol Off J Am Soc Clin Oncol. 2007:25:4575-80 14.

6. Vigano L, Ferrero A, Lo Tesoriere R, Capussotti L. Liver surgery for colorectal metastases: results after 10 years of follow-up. Long-term survivors, late recurrences, and prognostic role of morbidity. Ann Surg Oncol. 2008;15:2458-64.

7. Gillams AR, Lees WR. Five-year survival in 309 patients with colorectal liver metastases treated with radiofrequency ablation. Eur Radiol. 2009; 19:1206-13 22.

8. Siperstein AE, Berber E, Ballem N, Parikh RT. Survival after radiofrequency ablation of colorectal liver metastases,10-year experience. Ann Surg. 2007. 246:559-67.

9. Herfarth KK, Debus J, Wannenmacher M. Stereotactic single-dose radiation therapy of liver tumors: results of a phase I/II trial. J Clin Oncol. 2001:19:164-70 35.

10. Lee MT, Kim JJ, Dinniwell R, et al. Phase I study of individualized stereotactic body radiotherapy of liver metastases. J Clin Oncol. 2009;27:1585-91 36.

11. Goodman KA, Wiegner EA, Maturen KE, et al. Dose-escalation study of single-fraction stereotactic body radiotherapy for liver malignancies. Int J Radiat Oncol Biol Phys. 2010;78:486-93 37.

12. Rusthoven KE, Kavanagh BD, Cardenes H, et al. Multi-institutional phase I/II trial of stereotactic body radiation therapy for liver metastases. J Clin Oncol. 2009;27:1572-8 38.

13. Ambrosino G, Polistina F, Costantin G, et al. Image-guided robotic stereotactic radiosurgery for unresectable liver metastases: preliminary results. Anticancer Res. 2009:29:3381-4 39.

14. Mendez Romero A, Wunderink W, van Os RM, et al. Quality of life after stereotactic body radiation therapy for primary and metastatic liver tumors. Int J Radiat Oncol Biol Phys. 2008;70:1447-52 40.

15. Hoyer M, Roed H, Traberg HA, et al. Phase II study on stereotactic body radiotherapy of colorectal metastases. Acta Oncol. 2006;45:823-30.

16. Scorsetti M, Arcangeli S, Tozzi A, et al. Is stereotactic body radiation therapy an attractive option for unresectable liver metastasis? A preliminary report from a phase 2 trial. Int J Radiat Oncol Biol Phys. 2013;86:336-42.

17. Scorsetti M, Comito T, Tozzi A, Navarria P, Fogliata A, CLerici E, et al. Final results of a phase $\|$ trial for stereotactic body radiation therapy for patients with inoperable liver metastases from colorectal cancer. J Cancer Res Clin Concol. 2015;141:543-53.

18. Chang DT, Swaminath A, Kozak M, Weintraub J, Koong AC, Kim J, Dinniwell R, Brierley J, Kavanagh BD, Dawson LA, Schefter TE. Stereotactic body radiotherapy for colorectal liver metastases. A pooled analysis. Cancer. 2011;117:4060-9.46.

19. Fode MM, Høyer M. Survival and prognostic factors in 321 patients treated with stereotactic body radiotherapy for oligo-metastases. Radiother Oncol. 2015;114:155-60.

20. Comito T, Cozzi L, Clerici E, et al. Stereotactic ablative radiotherapy (SABR) in inoperable oligometastatic disease from colorectal cancer: a safe and effective approach. BMC Cancer. 2014;14:619. 
21. Ben-Josef E, Lawrence TS. Using a bigger hammer: the role of stereotactic body radiotherapy in the management of oligometastases. J Clin Oncol. 2009;27:1537-9.

22. McCammon R, Schefter TE, Gaspar LE, Zaemisch R, Gravdahl D, Kavanagh B. Observation of a dose-control relationship for lung and liver tumors after stereotactic body radiation therapy. Int J Radiat Oncol Biol Phys. 2009;73:112-8.

23. Bae SH, Kim MS, Cho CK, et al. High dose stereotactic body radiotherapy using three fractions for colorectal oligometastases. J Surg Oncol. 2012;106:138-43.

24. Eisenhauer $E$, Therasse $P$, Bogaerts $J$, et al. New response evaluation criteria in solid tumors: revised RECIST guideline (version 1.1). Eur J Cancer. 2009;45:228-47.

25. Adams RB, Aloia TA, Loyer E, Pawlik TM, Taouli B, Vauthey JN. Selection for hepatic resection of colorectal liver metastases: expert consensus statement. HPB. 2013;15:91-103.

26. Meyer JJ, Foster RD, Lev-Cohain N, et al. A phase I dose-escalation trial of single-fraction stereotactic radiation therapy for liver metastases. Ann Surg Oncol. 2016;23:218-24.

27. Toesca DA, Osmundson EC, Eyben RV, et al. Central liver toxicity after SBRT: an expanded analysis and predictive nomogram. Radiother Oncol. 2017;122:130-6.

28. Van Cutsem E, Cervantes A, Adam R, et al. ESMO consensus guidelines for the management of patients with metastatic colorectal cancer. Ann Oncol. 2016;27:1386-422.

29. Scorsetti M, Comito T, Cozzi L, Clerici E, Tozzi A, Franzese C, et al. The challenge of inoperable hepatocellular carcinoma (HCC): results of a single institutional experience on stereotactic body radiation therapy (SBRT). J Cancer Res Clin Oncol. 2015;141:1301-9.

30. McPartlin A, Swaminath A, Wang R, et al. Long-term outcomes of phase 1 and 2 studies of SBRT for hepatic colorectal metastases. Int J Radiat Oncol Biol Phys. 2017;99:388-95.

31. Joo JH, Park JH, Kim JC, et al. Local control outcomes using stereotactic body radiation therapy for liver metastases from colorectal cancer. Int J Radiat Oncol Biol Phys. 2017:99:876-83.

32. Klement RJ, Guckenberger M, Alheid H, et al. Stereotactic body radiotherapy for oligo-metastatic liver disease - influence of pre-treatment chemotherapy and histology on local tumor control. Radiother Oncol. 2017;123:227-33.

33. Klement RJ. Radiobiological parameters of liver and lung metastases derived from tumor control data of 3719 metastases. Radiother Oncol. 2017;123:218-26.

34. Ahmed KA, Caudell JJ, El-Haddad G, et al. Radiosensitivity differences between liver metastases based on primary histology suggest implications for clinical outcomes after stereotactic body radiation therapy. Int J Radiat Oncol Biol Phys. 2016;95:1399-404.

35. Oishi N, Yamashita T, Kaneko S. Molecular biology of liver cancer stem cells. Liver Cancer. 2014;3:71-84.

36. Fong Y, Fortner J, Sun RL, Brennan MF, Blumgart LH. Clinical score for predicting recurrence after hepatic resection for metastatic colorectal cancer: analysis of 1001 consecutive cases. Ann Surg. 1999;230:309e18.

37. Dhir M, Lyden ER, Wang A, Smith LM, Ullrich F, Are C. Influence of margins on overall survival after hepatic resection for colorectal metastasis: a metaanalysis. Ann Surg. 2011;254:234e42.

38. Nordlinger B, Sorbye H, Glimelius B, et al. Perioperative chemotherapy with FOLFOX4 and surgery versus surgery alone for resectable liver metastases from colorectal cancer (EORTC intergroup trial 40983): a randomized controlled trial. Lancet. 2008;371:1007-16.

39. Fairhurst K, Leopardi L, Satyadas T, Maddern G. The safety and effectiveness of liver resection for breast cancer liver metastases: a systematic review. Breast. 2016;30:175-84.

40. Adam R, Chiche L, Aloia T, et al. Hepatic resection for non-colorectal nonendocrine liver metastases: analysis of 1452 patients and development of a prognostic model. Ann Surg. 2006;244:524-35.

41. Goodman B, Mannina E, Althouse S, Maluccio M, Cárdenes H. Long-term safety and efficacy of stereotactic body radiation therapy for hepatic oligometastases. Pract Radiat Oncol. 2016;6:86-95.

42. Méndez Romero A, Keskin-Cambay F, van Os RM, et al. Institutional experience in the treatment of colorectal liver metastases with stereotactic body radiation therapy. Rep Pract Oncol Radiother. 2017;22:126-31.

43. Mazzola R, Fersino S, Alongi P, Di Paola F, Gregucci F, Aiello D, et al. Stereotactic body radiation therapy for liver oligometastases: predictive factors of local response by 18F-FDG-PET/CT. Br J Radiol. 2018:91:20180058.

44. Innat P, Skacelikova E, Tesar M, Penka I. Stereotactic body radiotherapy using the CyberKnife system in the treatment of patients with liver metastases: state of the art. Onco Targets. 2018;11:4685-91.

Ready to submit your research? Choose BMC and benefit from:

- fast, convenient online submission

- thorough peer review by experienced researchers in your field

- rapid publication on acceptance

- support for research data, including large and complex data types

- gold Open Access which fosters wider collaboration and increased citations

- maximum visibility for your research: over $100 \mathrm{M}$ website views per year

At $\mathrm{BMC}$, research is always in progress.

Learn more biomedcentral.com/submissions 